\title{
Identifying Inter-Project Relationships with Recurrent Neural Networks: Towards an AI Framework of Project Success Prediction
}

\author{
Ming-Wei Hsu ${ }^{a^{*}}$, Nicholas Dacre ${ }^{a}, \&$ PK Senyo ${ }^{a}$ \\ ${ }^{\text {a }}$ University of Southampton Business School, University of Southampton, Southampton, UK \\ ${ }^{*}$ Corresponding Author: M-W.Hsu@ soton.ac.uk
}

\begin{abstract}
A growing number of emerging studies have been undertaken to examine the mediating dynamics between intelligent agents, activities, and cost within allocated budgets to predict the outcomes of complex projects in dint of their significant uncertain nature in achieving a successful outcome. Emerging studies have used machine learning models to perform predictions, and artificial neural networks are the most frequently used machine learning model. However, most machine learning algorithms used in prior studies generally assume that input features, such as project complexity, team size and strategic importance, and prediction outputs, are independent. That is, a project's success is assumed to be independent of other projects. As the datasets used to train in prior studies often contain projects from different clients across industries, this theoretical assumption remains tenable. However, in practice, projects are often interrelated across several dimensions, such as distributed overlapping teams. Therefore, we argue that the inter-project relationships should be taken into consideration to improve prediction performance. Furthermore, an ongoing ethnographic study at a leading project management artificial intelligence consultancy, referred to in this research as Company Alpha, suggests that projects within the same portfolio frequently share overlapping characteristics. To capture the emergent inter-project relationships, this study aims to compare two specific types of artificial neural network prediction performances; (i) multilayer perceptron and; (ii) recurrent neural networks. The multilayer perceptron is one of the most widely used artificial neural networks in the project management literature, and recurrent networks are distinguished by the memory they take from prior inputs to influence input and output. Through this comparison, this research will examine whether recurrent neural networks can capture the potential inter-project relationship towards achieving improved performance in contrast to multilayer perceptron. Our empirical investigation using ethnographic practice-based exploration at Company Alpha will contribute to project management knowledge and support developing an intelligent project prediction AI framework with future applications for project practice.
\end{abstract}

Keywords: Project Management, Artificial Intelligence, Project Success, AI, Intelligent Project Prediction.

\section{Citation}

Hsu, M-W., Dacre, N., \& Senyo, P.K. (2021). Identifying Inter-Project Relationships with Recurrent Neural Networks: Towards an AI Framework of Project Success Prediction. British Academy of Management, Lancaster, UK 


\section{Introduction}

Project failure fundamentally remains one of the most pressing challenges for the project profession, and an area of critical and ongoing investigation in related academia (Kappelman et al., 2006; Sage et al., 2014). Extant research has focused a considerable amount of attention on project success and failure, and associated project outcomes. However, in practice this remains a stagnant challenge project profession. Studies on average suggest that around $80 \%$ of all projects fail to wholly achieve their planned outcomes within the constraints of time, cost, and scope, without realising their full anticipated benefits(Lenfle \& Loch, 2017).

As projects are ubiquitous across all industries, a pressing scholarly and practice-based requirement is required to continue in this critical vein of research (Dacre et al., 2019). Identifying the projects that are likely to fail allows in-time management intervention and is considered important in preventing project failure (Ivory \& Alderman, 2005). Recent technological innovations have enabled a more significant amount of attention to applying artificial intelligence in mitigating, or alleviating potential project failures (Dacre et al., 2020). Coupled with the greater use of machine learning, the project profession is currently undergoing a paradigm shift across our understanding of these technologies and their implementations for current and future challenges (Brookes et al., 2020). There has been growing suggestions that these technologies can increase the rates of project success. This inherently leads to a growing rise in the application of AI techniques in project management (Dacre et al., 2020; Wen et al., 2012).

In the extant literature where prior studies have applied artificial intelligence and machine learning to predict project success, these have implemented various algorithmic models such as through the use of artificial neural networks (de Barcelos Tronto et al., 2008), support vector machines (Pospieszny et al., 2018), a model with genetic algorithm (Ko Chien-Ho \& Cheng Min-Yuan, 2007), and decision trees (Gondia Ahmed et al., 2020). While these studies have hitherto provided some academically valuable insights, they are primarily positioned within the fundamental assumption that inputs and outputs are independent.

This approach inherently offers a proposition that suggests that a project's successful outcome is independent of its broader program context and inter-project dependencies. However, datasets are implemented in the application and training of these models encompass data across a range of different projects through a cross-section of industries, in dint of the fact that projects can be interrelated, either directly or indirectly, and therefore may rely on and share common project-based resources. Therefore, in this research we propose the argument that the inter-project relationship is both critical and vital in achieving improved performance towards successful project outcomes. In this vein, this study proposes implementing recurrent neural networks that facilitate the application of short-term memory in neurons by delivering the outputs of a layer to the stage of the input (Donkers et al., 2017).

The overarching aim of this study is to capture the effects of inter-project relationships in project success prediction, by comparing the performance of two types of artificial neural networks. The first one being multilayer perception, and the second is recurrent neural 
networks. The ensuing sections of this research, presents a critical review of machine learning within the context of project management, to further explicate gaps in prior literature that underpin the current research premise. Further to this, the study outlines the research methodology supported by discussing the data and measures undertaken as part of the ongoing ethnographic style study. Finally, this approach uncovers the application of both basic and advanced artificial neural networks in or church der to address the overarching research aim.

\section{Inter-Project Relationships}

Emerging studies implement machine learning to predict the efforts, cost and outcomes of projects. Wen et al. (2012) reviewed 84 studies using machine learning algorithms for project effort estimation and found that artificial neural networks were among the most widely used models. Ling and Liu (2004) used a backpropagation neural network to predict the performance of construction projects. The study relied on data sets of 33 projects, including engagement with 32 respondents across the industry. Berlin et al. (2009) used artificial networks and linear regression to estimate project effort group International Software Benchmarking Standards Group (ISBSG) dataset. This represents one of the largest project databases for undertaking research in this field, and findings suggested that official neural networks generally outperformed linear regression models.

Additionally, de Barcelos Tronto et al. (2008) found that artificial neural networks performed better than multiple regression models in project effort estimation using the Constructive Cost Model (COCOMO) dataset previously published by Boehm (1984) underpinned by research across 63 projects. López-Martín and Abran (2015) compared the performance of a multilayer feedforward neural network model, also called a multilayer perceptron, and a radial basis function neural network model in project effort estimation with the ISBSG dataset. The accuracy of machine learning models is arguably more sustained than statistical methods.

A smaller number of studies have proposed hybrid models by combining multiple machine learning models. For example, Ko \& Cheng (2007) used a hybrid approach that fuses genetic algorithms, fuzzy logic, and neural networks to predict project success with the Continuous Assessment of Project Performance (CAPP) database across 54 projects. They calculate project success as a ratio of spent resource to planned resource, which is similar to Cheng et al. (2010)'s calculation of project success.

There are very few studies that do not include artificial neural networks. One exception is that Gondia Ahmed et al. (2020) implemented a decision tree and naïve Bayesian classification algorithms to predict potential delays in construction projects. The research was carried out across a dataset of 51 construction projects stemming from 28 different organisations. The two algorithms were selected mainly because they are suited to smallsized data sets. The following table summarises the models implemented across these studies. 


\begin{tabular}{|c|c|c|c|c|}
\hline Study & Prediction & Algorithm & ANN Type & Data \\
\hline (Pospieszny et al., 2018) & $\begin{array}{l}\text { Software } \\
\text { effort }\end{array}$ & $\begin{array}{l}\text { SVM, ANN, linear } \\
\text { model }\end{array}$ & MLP & ISBSG \\
\hline $\begin{array}{l}\text { (de Barcelos Tronto et } \\
\text { al., 2008) }\end{array}$ & $\begin{array}{l}\text { Software } \\
\text { effort }\end{array}$ & $\begin{array}{l}\text { ANN } \\
\text { linear model }\end{array}$ & MLP & COCOMO \\
\hline $\begin{array}{l}\text { (López-Martín \& Abran, } \\
\text { 2015) }\end{array}$ & $\begin{array}{l}\text { Software } \\
\text { duration }\end{array}$ & $\begin{array}{l}\text { ANN } \\
\text { linear }\end{array}$ & $\begin{array}{l}\text { MLP; } \\
\text { Radial basis } \\
\text { function } \\
\text { neural } \\
\text { network } \\
\text { (RBFNN) }\end{array}$ & ISBSG \\
\hline (Berlin et al., 2009) & $\begin{array}{l}\text { Software } \\
\text { duration, } \\
\text { cost }\end{array}$ & $\begin{array}{l}\text { ANN } \\
\text { linear }\end{array}$ & $\begin{array}{l}\text { MLP with } 1 \\
\text { and } 2 \text { hidden } \\
\text { layers }\end{array}$ & ISBSG \\
\hline (Ling \& Liu, 2004) & Cost, time & ANN & $\begin{array}{l}\text { MLP with } \\
\text { one or more } \\
\text { hidden } \\
\text { layers }\end{array}$ & Own survey \\
\hline (Cheng et al., 2010) & success & $\begin{array}{l}\text { support vector machine } \\
\text { (SVM) } \\
\text { fast messy genetic } \\
\text { algorithm (fmGA) }\end{array}$ & $\mathrm{n} / \mathrm{a}$ & CAPP \\
\hline $\begin{array}{l}\text { (Ko Chien-Ho \& Cheng } \\
\text { Min-Yuan, 2007) }\end{array}$ & success & $\begin{array}{l}\text { genetic algorithms } \\
\text { (GAs), fuzzy logic (FL), } \\
\text { and neural networks } \\
(\mathrm{NNs})\end{array}$ & MLP & CAPP \\
\hline $\begin{array}{l}\text { (Gondia Ahmed et al., } \\
\text { 2020) }\end{array}$ & Delay & $\begin{array}{l}\text { decision tree }(\mathrm{DT}) \\
\text { naïve Bayesian } \\
\text { classification }(\mathrm{NB})\end{array}$ & $\mathrm{n} / \mathrm{a}$ & Own survey \\
\hline
\end{tabular}

The emergent theme across the number of different studies included in the above analysis, suggests a strong propensity for using multilayer perceptron inherently derived from the assumption that projects are independent of each other and the larger program context. However, this may not hold true in project management. We argue that some projects interrelate more than other projects.

Specifically, the projects managed by the same team are likely to relate more than random projects, and a management team may learn from their experience to deliver more successful projects. The short-term memory characteristic of recurrent neural networks can be used to recognise the inter-project relationship which cannot be recognised with MLP commonly used in the literature (López-Martín \& Abran, 2015; Pospieszny et al., 2018). 


\section{Research Context}

The project dataset applied in this research is captured by adopting an ongoing ethnographic style research approach with a leading project management artificial intelligence consultancy, referred to by the pseudonym Company Alpha. The organisation is a high-profile industry leader in advanced artificial intelligence and data analytics for use across a range of project management processes. Several different input variables will be derived from ongoing concurrent project information, such as budgets, milestones, and stages, with the acute output variable being the successful outcome of projects.

The ethnographic approach to this research study enables the close identification and engagement of project variables and developments across the life cycle. Furthermore, this facilitates ongoing and consistent access to data. Therefore, project success is identified at the point when both time and resources criteria are achieved. In essence, this is identified when a project is delivered on time and within budget.

\section{Analysis}

In order to test the prediction accuracy of machine learning models, we divided a dataset into three non-overlapping sets for model training, validation, and testing. We used the training set to estimate the parameters of the model and use the validation set to tune metaparameters, such as the number of neurons in concealed layers. Therefore, we obtained a fully specified model with fixed meta-parameters based upon the union of the training and validation sets.

Subsequently, the fully-specified model was applied to the test set to generate predictions, and the predictions were compared to the actual values of the output variable in the test set to measure prediction accuracy. This approach has hitherto enabled us to mitigate potential overfitting problems to compare models in terms of their predictive accuracy.

We will include two machine learning algorithms in the analysis: multilayer perceptron and recurrent neural networks. Multilayer perceptron is one of the basic types of artificial neural networks extensively used across different settings (Hsu et al., 2016; Pospieszny et al., 2018). A multilayer perceptron neural network consists of input, hidden and output layers, where each layer has multiple information processing units referred to as neurons. The neurons of one layer are fully connected to the neurons of the next layer.

We followed the standard approach for binary classification problems, in that neuron in the input layer represent the original input variables, and the single neuron in the output layer represents the output of the model (Hastie et al., 2009). There are meta-parameters for the multilayered perceptron models, including the regularisation term such as the number of neurons in the hidden layer.

A grid search was used to search for suitable values for the meta-parameters (Cherkassky \& Ma, 2004). Candidate values for each meta-parameter were selected based on recommendations from the literature (Berry \& Linoff, 1997; Xu \& Chen, 2008), and we assessed all possible combinations of meta-parameter settings with empirical data. The network structure of our multilayered perceptron model is illustrated in Figure 1. 


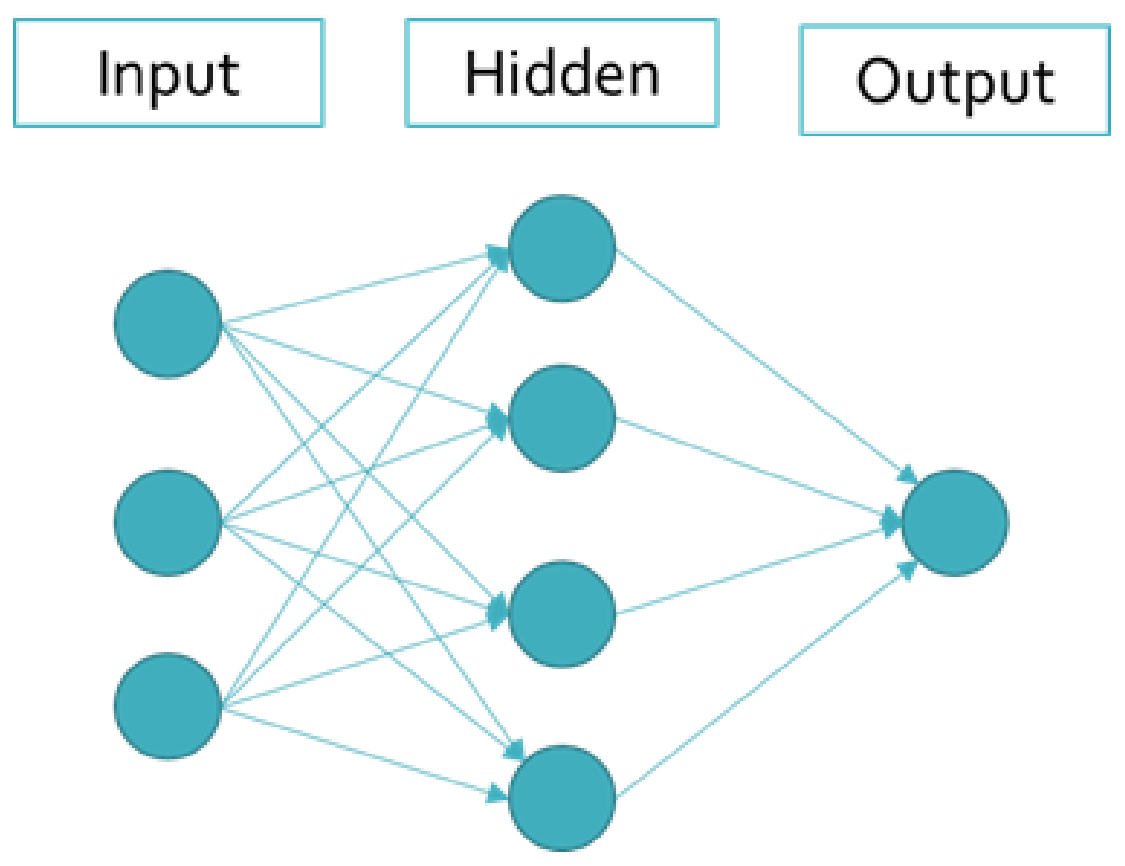

Figure 1: A 3 Layer Structure of a Multilayer Perceptron Neural Network

\section{Conclusion \& Further Research}

A recurrent neural network is a type of artificial neural network, with the substantial difference between a multilayered perceptron and a recurrent neural network is that the output of a particular layer in the latter is saved and driven back to the input (Sak et al., 2014). The cyclic connections in recurrent neural networks have been demonstrated to be a powerful approach to model sequence data in contrast to multilayered perceptron, and the application of recurrent neural networks in sequence labelling and towards prediction tasks, such as handwriting recognition and language modelling. As such, these are gaining in popularity (Donkers et al., 2017).

A recurrent neural network's input layer is equal to a multilayered perceptron, with each neuron in the next layer, such as the hidden layer, storing the information from a previous time point. That is, the cycles allow neurons to act as memory cells to store information in the network's internal states. Therefore, recurrent neural networks, in principle, can retain long-term temporal contextual information. Furthermore, this mechanism allows recurrent neural networks to exploit a dynamically changing contextual window over the input sequence history, rather than a static one implemented through the use of a multilayer perceptron (Donkers et al., 2017).

The ongoing project data collected at Company Alpha across several companies reveals that data is held in different formats and will need to be further examined, ratified, merged and processed for model training. Ensuing steps as part of this ongoing study will require developing and implementing a prototype system reliant on multilayer perceptron and recurrent neural networks to predict potential project successes. This approach will sustain the comparison and evaluation prediction performance of multilayered perceptron and recurrent networks, with the results analysed to further guide the development of an AI framework for project success reliant on algorithmic machine learning. 


\section{References}

B. W. Boehm. (1984). Software Engineering Economics. IEEE Transactions on Software Engineering, SE-10(1), 4-21. https://doi.org/10.1109/TSE.1984.5010193

Berlin, S., Raz, T., Glezer, C., \& Zviran, M. (2009). Comparison of estimation methods of cost and duration in IT projects. Information and Software Technology, 51(4), 738748. https://doi.org/10.1016/j.infsof.2008.09.007

Berry, M. J., \& Linoff, G. (1997). Data mining techniques: For marketing, sales, and customer support. John Wiley \& Sons, Inc.

Brookes, N., Lattuf Flores, L., Dyer, R., Stewart, I., Wang, K., \& Dacre, N. (2020).

Project Data Analytics: The State of the Art and Science [APM Research]. Association for Project Management (APM). https://doi.org/10.5281/zenodo.4621177

Cheng, M.-Y., Wu, Y.-W., \& Wu, C.-F. (2010). Project success prediction using an evolutionary support vector machine inference model. 25th International Symposium on Automation and Robotics in Construction, 19(3), 302-307.

https://doi.org/10.1016/j.autcon.2009.12.003

Cherkassky, V., \& Ma, Y. (2004). Practical selection of SVM parameters and noise estimation for SVM regression. Neural Networks, 17(1), 113-126.

https://doi.org/10.1016/S0893-6080(03)00169-2

Dacre, N., Kockum, F., \& Senyo, P. (2020). Transient Information Adaptation of Artificial Intelligence: Towards Sustainable Data Processes in Complex Projects. British Academy of Management (BAM), Manchester, UK.

http://dx.doi.org/10.2139/ssrn.3813559

Dacre, N., Senyo, P., \& Reynolds, D. (2019). Is an Engineering Project Management Degree Worth it? Developing Agile Digital Skills for Future Practice. Engineering Education Research Network (EERN), Coventry, UK.

http://dx.doi.org/10.2139/ssrn.3812764

de Barcelos Tronto, I. F., da Silva, J. D. S., \& Sant'Anna, N. (2008). An investigation of artificial neural networks based prediction systems in software project management. Selected Papers from the 2006 Brazilian Symposia on Databases and on Software Engineering, 81(3), 356-367. https://doi.org/10.1016/j.jss.2007.05.011

Donkers, T., Loepp, B., \& Ziegler, J. (2017). Sequential User-based Recurrent Neural Network Recommendations. In Proceedings of the Eleventh ACM Conference on Recommender Systems (pp. 152-160). Association for Computing Machinery. https://doi.org/10.1145/3109859.3109877

Gondia Ahmed, Siam Ahmad, El-Dakhakhni Wael, \& Nassar Ayman H. (2020). Machine Learning Algorithms for Construction Projects Delay Risk Prediction. Journal of Construction Engineering and Management, 146(1), 04019085. https://doi.org/10.1061/(ASCE)CO.1943-7862.0001736

Hastie, T., Tibshirani, R., \& Friedman, J. (2009). The elements of statistical learning. Springer. 
Hsu, M.-W., Lessmann, S., Sung, M.-C., Ma, T., \& Johnson, J. E. V. (2016). Bridging the divide in financial market forecasting: Machine learners vs. Financial economists. Expert Systems with Applications, 61, 215-234. https://doi.org/10.1016/j.eswa.2016.05.033

Ivory, C., \& Alderman, N. (2005). Can Project Management Learn Anything from Studies of Failure in Complex Systems? Project Management Journal, 36(3), 5-16. https://doi.org/10.1177/875697280503600302

Kappelman, L. A., McKeeman, R., \& Zhang, L. (2006). Early warning signs of IT project failure: The dominant dozen. Information Systems Management, 23(4), 31-36.

Ko Chien-Ho \& Cheng Min-Yuan. (2007). Dynamic Prediction of Project Success Using Artificial Intelligence. Journal of Construction Engineering and Management, 133(4), 316-324. https://doi.org/10.1061/(ASCE)0733-9364(2007)133:4(316)

Lenfle, S., \& Loch, C. (2017). Has megaproject management lost its way. The Oxford Handbook of Megaproject Management, 21-38.

Ling, F. Y. Y., \& Liu, M. (2004). Using neural network to predict performance of design-build projects in Singapore. Building and Environment, 39(10), 1263-1274. https://doi.org/10.1016/j.buildenv.2004.02.008

López-Martín, C., \& Abran, A. (2015). Neural networks for predicting the duration of new software projects. Journal of Systems and Software, 101, 127-135.

https://doi.org/10.1016/j.jss.2014.12.002

Pospieszny, P., Czarnacka-Chrobot, B., \& Kobylinski, A. (2018). An effective approach for software project effort and duration estimation with machine learning algorithms. Journal of Systems and Software, 137, 184-196. https://doi.org/10.1016/j.jss.2017.11.066

Sage, D., Dainty, A., \& Brookes, N. (2014). A critical argument in favor of theoretical pluralism: Project failure and the many and varied limitations of project management. International Journal of Project Management, 32(4), 544-555.

Sak, H., Senior, A. W., \& Beaufays, F. (2014). Long short-term memory recurrent neural network architectures for large scale acoustic modeling. Google.

http://research.google/pubs/pub43905.pdf

Wen, J., Li, S., Lin, Z., Hu, Y., \& Huang, C. (2012). Systematic literature review of machine learning based software development effort estimation models. Information and Software Technology, 54(1), 41-59. https://doi.org/10.1016/j.infsof.2011.09.002

$\mathrm{Xu}, \mathrm{S} .$, \& Chen, L. (2008). A novel approach for determining the optimal number of hidden layer neurons for FNN's and its application in data mining. 5th International Conference on Information Technology and Applications (ICITA 2008), Cairns, Queensland, Australia. 